\title{
On the Length of Shortest 2-Collapsing Words
}

\author{
Alessandra Cherubini ${ }^{1}$ and Andrzej Kisielewicz ${ }^{2}$ and Brunetto Piochi ${ }^{3} \|$ \\ ${ }^{1}$ Politecnico di Milano, Dipart. di Matematica "F. Brioschi”, Piazza L. da Vinci 32 - 20133 Milano, Italy \\ alessandra.cherubiniepolimi.it \\ ${ }^{2}$ University of Wroctaw, Institute of Mathematics, pl. Grunwaldzki 2 - 50-384 Wroctaw, Poland \\ kisielemath.uni.wroc.pl \\ ${ }^{3}$ Università di Firenze, Dipart. di Matematica "U. Dini”, viale Morgagni 67/a - 50134 Firenze, Italy; \\ piochiemath. unifi.it
}

received May 12, 2008, revised October 24, 2008, accepted December 10, 2008.

Given a word $w$ over a finite alphabet $\Sigma$ and a finite deterministic automaton $\mathcal{A}=\langle Q, \Sigma, \delta\rangle$, the inequality $|\delta(Q, w)| \leq$ $|Q|-k$ means that under the natural action of the word $w$ the image of the state set $Q$ is reduced by at least $k$ states. The word $w$ is $k$-collapsing ( $k$-synchronizing) if this inequality holds for any deterministic finite automaton (with $k+1$ states) that satisfies such an inequality for at least one word. We prove that for each alphabet $\Sigma$ there is a 2collapsing word whose length is $\frac{|\Sigma|^{3}+6|\Sigma|^{2}+5|\Sigma|}{2}$. Then we produce shorter 2-collapsing and 2-synchronizing words over alphabets of 4 and 5 letters.

Keywords: property $\Delta_{k}$, finite automata, synchronizing finite automata

\section{Introduction and preliminaries.}

In this paper by an automaton $\mathcal{A}=\langle Q, \Sigma, \delta\rangle$ we mean a finite deterministic automaton with state set $Q$, input alphabet $\Sigma$, and transition function $\delta: Q \times \Sigma \rightarrow Q$. The action of $\Sigma$ on $Q$ given by $\delta$ will be denoted by concatenation: $q a=\delta(q, a)$. This action extends naturally, by composition, to the action of the words of $\Sigma^{*}$ on $Q$. Given a word $w \in \Sigma^{*}$, we will be interested in the difference of the cardinalities $|Q|-|Q w|$. This difference is called the deficiency of the word $w$ with respect to $\mathcal{A}$ and will be denoted $\operatorname{df}_{\mathcal{A}}(w)$.

For a fixed $k \geq 1$, a word $w \in \Sigma^{*}$ is called $k$-compressing for $\mathcal{A}$ if $\operatorname{df}_{\mathcal{A}}(w) \geq k$. An automaton $\mathcal{A}$ is $k$-compressible if there exists a $k$-compressing word for $\mathcal{A}$. A word $w \in \Sigma^{*}$ is $k$-collapsing (over $\Sigma$ ) if it is $k$-compressing for every $k$-compressible automaton with the input alphabet $\Sigma$. A word $v$ is called

\footnotetext{
${ }^{\dagger}$ This research was partially supported by the ESF programme Automatha 1365-8050 @ 2009 Discrete Mathematics and Theoretical Computer Science (DMTCS), Nancy, France
} 
$k$-synchronizing if it is $k$-compressing for all $k$-compressible automata with $k+1$ states. Of course a $k$-collapsing word is also $k$-synchronizing.

The concept of a $k$-collapsing word is a natural automata-theoretic interpretation of the notion of words with the property $\Delta_{k}$ introduced for algebraic motivations in [12]. Actually $k$-collapsing words can be seen as universal testers for checking whether an automaton is $k$-compressible. They are a blackbox versions of words considered in the so called generalized Černý conjecture that extends the celebrated Černý conjecture [5]. Recall that the former conjecture deals with the length of the shortest $k$-compressing word for a given $k$-compressible automaton. In [12] it was proved that $k$-collapsing words always exist, for each $\Sigma$ and each $k \geq 1$, by means of a recursive construction which gives a word whose length is doubly exponential as function of $k$. Then better lower and upper bounds for the length $c(k, t)$ of the shortest $k$-collapsing words on an alphabet of cardinality $t$ were given in [9] and the lower bound for the case $k=2$ was slightly improved in [10]. So far for the case $k=2$ the best known bounds are the following: $2 t^{2} \leq c(2, t) \leq t^{3}+3 t^{2}+2 t$.

In [1, 2], two different algorithms for deciding whether a word is 2-collapsing are given and another more combinatorial approach to the same problem is presented in [8]. Here we get a better upper bound for $c(2, t)$, namely $c(2, t) \leq \frac{t^{3}+6 t^{2}+5 t}{2}$, by means of the latter approach. Moreover we prove that this bound can be improved at least for small values of $t$ : we build, again by using this approach, shorter 2-collapsing words on alphabets of cardinality 4 and 5. The reader is referred to [9] and to [4] for some further references and connections to some topics in Theoretical Computer Science, Language Theory and Combinatorics. Here for sake of completeness we shortly recall notation and main properties.

We view an automaton $\mathcal{A}=\langle Q, \Sigma, \delta\rangle$ as a set of transformations on $Q$ labelled by letters of $\Sigma$ rather than as a standard triple. By transformations of $\mathcal{A}$ we mean those transformations on $Q$ that are induced via $\delta$ by letters of $\Sigma$. In order to define an automaton it is enough just to assign to every letter of $\Sigma$ a transformation on $Q$. Now, for $a \in \Sigma, \mathrm{df}_{\mathcal{A}}(a)=0$ if and only if the corresponding transformation is a permutation on $Q$. If $\operatorname{df}_{\mathcal{A}}(a)=1$, then there is a state $z \in Q$ which does not belong to the image $Q a$ and two different states $x, y \in Q$ satisfying $x a=y a$; in such a case the corresponding transformation will be referred to as a transformation of type $\{x, y\} \backslash z$ ( $x, y$ have the same image under the transformation, $z$ is missed). The semigroup generated by the transformations of $\mathcal{A}$ consists precisely of the transformations corresponding to words in $\Sigma^{*}$. It contains a group generated by those transformations that are permutations; this group is called the group of permutations of $\mathcal{A}$.

It is well known ([9]) that each 2-collapsing word over a fixed alphabet $\Sigma$ is 2-full, i.e. it contains any word of length 2 on $\Sigma$ among its factors, hence to characterize 2-collapsing words it is enough to consider 2 -full words compressing each 2-compressible automata such that no word of length 2 is 2 -compressing for them (proper 2-compressible automata, for short). Proper 2-compressible automata must have both non-permutation and permutation transformations and they are characterized by the following:

Proposition 1 ([8]) An automaton $\mathcal{A}$ is proper 2-compressible if and only if $\mathcal{A}$ fulfils one of the following conditions:

1. there are two states $x, y$ such that all non-permutation transformations are of the same type $\{x, y\} \backslash x$, and the group of permutations fixes neither the element $x$ nor the set $\{x, y\}$;

2. there is a state $x$ such that each non-permutation transformation is of type $\{x, z\} \backslash x$ for some state $z$, at least two different types occur, and the group of permutations does not fix $x$; 
3. there are two states $x, y$ such that each non-permutation transformation is of type $\{x, y\} \backslash x$ or $\{x, y\} \backslash y$, both types occur, and the group of permutations does not fix the set $\{x, y\}$.

Automata of the first two types are called MONO-automata, of the latter one are called STEREOautomata.

In [8] it is proved that a word $w \in \Sigma^{*}$ is 2-collapsing if and only if certain systems of conditions on permutations have only trivial solutions. We recall some notations from [6]. In the sequel, as no confusion can raise, we view the set $Q$ of the states of $\mathcal{A}$ as a set of natural numbers: $Q=\{1,2, \ldots, n\}$, so that permutations on $Q$ can be viewed as elements of the symmetric group $\operatorname{Sym}_{n}$. The two states $x, y$ occurring in Proposition 1 will be then denoted by 1 and 2. By a role assignment we mean an arbitrary partition of the alphabet $\Sigma$ with a distinguished nonempty block $\Pi \subset \Sigma$. Roughly speaking letters in $\Pi$ play the role of permutation letters of $\Sigma$ and the remaining letters play the role of non-permutation letters. Let $\Upsilon=\Sigma \backslash \Pi$. Since non-permutation letters in proper 2-compressible automata can be of different types, a role assignment induces on $\Upsilon$ a further partition in non empty blocks. We denote this partition either as $\left\{D_{2}, \ldots, D_{h}\right\}, h \geq 2$, where letters in $D_{i}$ will play the role of the non-permutation letters of the form $\{1, i\} \backslash 1$ or as $\left\{E_{1}, E_{2}\right\}$ where letters in $E_{1}, E_{2}$ will play the role of non-permutation letters of the form $\{1,2\} \backslash 1$ and $\{1,2\} \backslash 2$ respectively. In the first case the partition of $\Sigma$ will be called a MONO-role assignment (DB-partition in [8]) and in the latter a STEREO-role assignment (3DB-partition in [8]).

Let $(\Pi, \Upsilon)$ be an arbitrary role assignment, then each word $w \in \Sigma^{+}$can be uniquely represented in the following form:

$$
w=u_{0} p_{1} u_{1} \ldots u_{m-1} p_{m} u_{m}
$$

where $p_{1}, \ldots, p_{m} \in \Pi^{+}, u_{1}, \ldots, u_{m-1} \in \Upsilon^{+}, u_{0}, u_{m} \in \Upsilon^{*}$ and $m$ is a positive integer. We say that a factor $p_{i}$ of the decomposition (1) is an inner factor if both $u_{i-1}$ and $u_{i}$ are non-empty. Then for a MONO-role assignment $(\Pi, \Upsilon)$ for each $a \in \Upsilon$ we denote by $S_{a}$ the set of the inner factors $p_{i}$ of $w$ such that $a$ is the first letter in $u_{i}$. Otherwise if $(\Pi, \Upsilon)$ is a STEREO-role assignment $\left(\Pi,\left\{E_{1}, E_{2}\right\}\right)$ we denote by $S_{k}, k=1,2$, the sets of the inner factors $p_{i}$ of $w$ such that the last letter of $u_{i-1}$ belongs to $E_{k}$.

We assign to the word $w \in \Sigma^{+}$, for each role assignment and for each inner factor $p_{i}$, a permutation condition of the form:

$-1 p_{i} \in\{1, j\}$ if the role assignment is MONO and $p_{i} \in S_{a}$ with $a \in D_{j}$,

- $k p_{i} \in\{1,2\}, k=1,2$, if the role assignment is STEREO and $p_{i} \in S_{k}$.

Then each role assignment associates to $w$ a system of permutation conditions formed by all the permutation conditions corresponding to the inner factors of $w$. In case of a MONO-role assignment $(\Pi, \Upsilon)$ such system will be denoted $\Gamma_{w}(\Pi, \Upsilon)$ otherwise it will be denoted $\Gamma_{w}^{\prime}(\Pi, \Upsilon)$.

Since letters of $\Pi$ are regarded as permutations acting on a finite set of positive integers $\{1,2, \ldots, n\}$ (representing $Q$ ), conditions of the form $j v \in A$ with $j$ positive integer, $v \in \Pi^{+}, A \subseteq\{1,2, \ldots, n\}$ mean that the image of $j$ under the product $v$ of permutations belongs to the set $A$. We say that a system of permutation conditions has a solution if there exists an assignment of permutations on a finite set $\{1,2, \ldots, n\}$ to letters in $\Pi$ such that all the conditions in the system are satisfied. Obviously the systems associated to $w$ by each role assignment have always the solution where all permutations are the identity. A solution of the system $\Gamma_{w}(\Pi, \Upsilon)$, associated to $w$ by a MONO-role assignment, is called trivial if all permutations fix 1. Also, in the special case when $\Upsilon$ consists of the unique block $D_{2}$ (and as a consequence, all $j$ 's on the right hand side of the conditions are equal to 2), a solution with all permutations fixing the set $\{1,2\}$ is considered trivial. The remaining solutions are nontrivial. Similarly a solution of the system 
$\Gamma_{w}^{\prime}(\Pi, \Upsilon)$, associated to $w$ by a STEREO-role assignment $(\Pi, \Upsilon)$ is called trivial if all the permutations fix $\{1,2\}$.

In [8] 2-collapsing words were characterized in terms of permutation conditions in the following way.

Theorem 2 ([8]) A word $w \in \Sigma^{+}$is 2-collapsing if and only if it is 2-full and the following conditions hold:

1. For each MONO-role assignment $(\Pi, \Upsilon)$ of $\Sigma$ all the solutions of the system $\Gamma_{w}(\Pi, \Upsilon)$ are trivial.

2. For each STEREO-role assignment $(\Pi, \Upsilon)$ of $\Sigma$ all the solutions of the system $\Gamma_{w}^{\prime}(\Pi, \Upsilon)$ are trivial.

By the above Theorem 2 one can readily derive the following characterization of 2 -synchronizing words.

Corollary $3 A$ word $w \in \Sigma^{+}$is 2-synchronizing if and only if it is 2-full and for each role assignment $(\Pi, \Upsilon)$ of $\Sigma$ all the solutions of the systems $\Gamma_{w}(\Pi, \Upsilon)$ (in case of MONO-role assignments) and $\Gamma_{w}^{\prime}(\Pi, \Upsilon)$ (in case of STEREO-role assignments) in the semigroup $\mathbf{T}_{3}$ of all transformations on 3 letters are trivial.

\section{Main result.}

We introduce some useful notations.

Definition 1 . A permutation $p$ on $\{1,2, \ldots, n\}$ is special with respect to the ordered pair $(i, j), i, j \in$ $\{1,2, \ldots, n\}$, whether it fixes either $\{i\}$ or $\{i, j\}$. Two permutations $p, z$ are special of the same type with respect to the ordered pair $(i, j)$ if both $p$ and $z$ fix $\{i\}$ or both of them fix $\{i, j\}$.

In the sequel by $(i) \ldots,(i j) \ldots,(i j h \ldots) \ldots$ we will denote respectively a permutation fixing $i$, containing a cycle $(i, j)$, containing a cycle $(i j h \ldots)$ of arbitrary length.

Note that the property of being special of the same type with respect to $(i, j)$ is not transitive; e.g. both the permutations $p=(1)(2 \ldots n)$ and $z=(12)(3 \ldots n)$ are special of the same type as $y=(1)(2)(3 \ldots n)$ with respect to $(1,2)$, but they are not of the same type. Note however that two special permutations $p$ and $z$ can be of the same type of $y$ with respect to $(i, j)$ without being of the same type only if one of them fixes $\{i\}$ and does not fix $j$, the other has a cycle $(i j)$ while $y$ fixes both $\{i\}$ and $\{j\}$. Trivial solutions of systems introduced before Theorem 2 are special of the same type with respect to $(1,2)$, so we are interested in sets of equations whose occurrence in a system guarantees that solutions are special of the same type with respect to $(1,2)$.

Lemma 4 Let $p, z$ be two permutations on $\{1,2, \ldots, n\}$ and let $i, j \in\{1,2, \ldots, n\}$. The permutations $p, z$ fulfil the conditions:

1. $i p \in\{i, j\}$

2. $i p^{2} \in\{i, j\}$

3. $i z \in\{i, j\}$

4. $i z^{2} \in\{i, j\}$

5. $i p z p \in\{i, j\}$ (or $i z p z \in\{i, j\})$ 
if and only if they are special of the same type with respect to $(i, j)$.

Proof: The "if part" is trivial. Conversely from condition 1. we immediately get that either $p$ fixes $i$ or it is of the form $p=(i j \ldots) \ldots$ and condition 2. gives that in such case $p=(i j) \ldots$ Similarly conditions 3 . and 4. give that $z$ either fixes $i$ or has the form $z=(i j) \ldots$ Now if $z=(i j) \ldots$ and $p$ fixes $i$ then $p$ fixes also $j$ otherwise condition 5. is not fulfilled. If $p=(i j) \ldots$ then $j z=i p z \in\{i, j\}$ by condition 5. whence $z$ fixes $\{i, j\}$.

Similarly, the following can be proved:

Lemma 5 Let $p, z$ be two permutations on $\{1,2, \ldots, n\}$ and let $i, j \in\{1,2, \ldots, n\}$. The permutations $p, z$ fulfil the conditions:

1. $i p \in\{i, j\}$

2. $i p^{2} \in\{i, j\}$

3. $i z^{2} \in\{i, j\}$

4. $i p z \in\{i, j\}$

5. $i z p \in\{i, j\}$

if and only if they are special of the same type with respect to $(i, j)$.

Theorem 6 The minimal 2-collapsing word on t letters has length less than or equal to

$$
\frac{t^{3}+6 t^{2}+5 t}{2}
$$

Proof: To show our bound we want to exhibit a 2-collapsing word, similar to the one introduced in [9]. Consider an alphabet $\Sigma=\left\{a_{1}, a_{2}, \ldots, a_{t}\right\}$ on $t$ letters in the lexicographic order and let $u=a_{1} a_{2} \ldots a_{t}$ and

$$
w=u\left(\prod_{q \in X} q u\right)
$$

where

$$
X=\left\{x, x^{2}, x y x: x, y \in \Sigma, y>x\right\} .
$$

We want to prove that the word $w$ is 2-collapsing. Let us consider different role assignments $(\Pi, \Upsilon)$ of $\Sigma$.

Assume that $(\Pi, \Upsilon)$ is a STEREO-role assignment with $\Upsilon=\left\{E_{1}, E_{2}\right\}$; let $h, k$ be respectively the least and the greatest index of an element in $\Upsilon$ and let $a_{j}$ be an element in $\Upsilon$ belonging to a different block than $a_{k}$ (eventually $h=j$ ). Each $a_{i}, i>k$ (if any) fixes $\{1,2\}$, since the factors $a_{k} a_{i} a_{k}, a_{j} a_{i} a_{j}$ occur in $w$ whence both the equations $1 a_{i} \in\{1,2\}$ and $2 a_{i} \in\{1,2\}$ occur in $\Gamma_{w}^{\prime}(\Pi, \Upsilon)$. More the product $a_{1} a_{2} \ldots a_{h-1}$ fixes $\{1,2\}$. In fact $a_{j}\left(a_{1} a_{2} \ldots a_{h-1}\right) a_{h}$ and $a_{k}\left(a_{1} a_{2} \ldots a_{h-1}\right) a_{h}$ are factors of $w$ and all $a_{1}, \ldots, a_{h-1}$ are in $\Pi$ hence both the equations $1\left(a_{1} a_{2} \ldots a_{h-1}\right) \in\{1,2\}$ and $2\left(a_{1} a_{2} \ldots a_{h-1}\right) \in\{1,2\}$ occur in $\Gamma_{w}^{\prime}(\Pi, \Upsilon)$. This yields that also any $a_{i}, i<h$ (if any) fixes $\{1,2\}$, since also $a_{i} a_{j} a_{i}\left(a_{1} a_{2} \ldots a_{h-1}\right) a_{h}$ and $a_{i} a_{k} a_{i}\left(a_{1} a_{2} \ldots a_{h-1}\right) a_{h}$ are factors of $w$ whence both the equations $1 a_{i}\left(a_{1} a_{2} \ldots a_{h-1}\right) \in\{1,2\}$ and $2 a_{i}\left(a_{1} a_{2} \ldots a_{h-1}\right) \in\{1,2\}$ occur in $\Gamma_{w}^{\prime}(\Pi, \Upsilon)$ with $a_{1} a_{2} \ldots a_{h-1}$ fixing $\{1,2\}$. Similarly, at last, each 
$a_{i}: h<i<k$ (if any) fixes $\{1,2\}$. In fact either $a_{h}$ and $a_{k}$ are in different blocks or there is an $a_{j} \in \Upsilon$ belonging to a different block with respect to $a_{h}$ and $a_{k}$, so for each $i, h<i<k$ there are two indices $s, r, h \leq s<i<r \leq k$ such that $a_{s}$ and $a_{r}$ are in different blocks of $\Upsilon$, hence $a_{i} a_{r} a_{i}\left(a_{1} a_{2} \ldots a_{h-1}\right) a_{h}$ and $a_{s} a_{i} a_{s}$ are factors of $w$.

Then let $(\Pi, \Upsilon)$ be a MONO-role assignment with $\Upsilon=\left\{D_{2}, D_{3}, \ldots D_{g}\right\}$ with $g>2$. Let $k$ be the greatest index such that $a_{k} \in \Upsilon$ then $a_{k} \in D_{k^{\prime}}$ for some $k^{\prime}$. Let $D_{j^{\prime}}$ be a block of $\Upsilon$ different from $D_{k^{\prime}}$ and let $a_{j} \in D_{j^{\prime}}$. We will prove that every $a_{i} \in \Pi$ fixes $\{1\}$. In fact if $i>k$ then $a_{k} a_{i} a_{k}, a_{j} a_{i} a_{j}$ are factors of $w$ hence both the equations $1 a_{i} \in\left\{1, k^{\prime}\right\}, 1 a_{i} \in\left\{1, j^{\prime}\right\}$ occur in the system $\Gamma_{w}(\Pi, \Upsilon)$, whence $a_{i}$ fixes $\{1\}$ for all $a_{i} \in \Pi$ with $i>k$, if any. Now let $i<k$. If $j<i<k$ then $a_{j} a_{i} a_{j}, a_{k} a_{k+1} \ldots a_{t} a_{i} a_{k} a_{i}$ are factors of $w$, if $i<j$ then $a_{k} a_{k+1} \ldots a_{t} a_{i} a_{j} a_{i}, a_{k} a_{k+1} \ldots a_{t} a_{i} a_{k} a_{i}$ are factors of $w$ and all elements $a_{k+1}, \ldots, a_{t}$ are in $\Pi$, hence they fix $\{1\}$. Then in the system $\Gamma_{w}(\Pi, \Upsilon)$ there are either the equations $1 a_{i} \in\left\{1, j^{\prime}\right\}$ and $1 a_{k+1} \ldots a_{t} a_{i} \in\left\{1, k^{\prime}\right\}$ or $1 a_{k+1} \ldots a_{t} a_{i} \in\left\{1, j^{\prime}\right\}$ and $1 a_{k+1} \ldots a_{t} a_{i} \in\left\{1, k^{\prime}\right\}$ from which in both cases we get that $1 a_{i} \in\left\{1, k^{\prime}\right\}$ and $1 a_{i} \in\left\{1, j^{\prime}\right\}$, thus all $a_{i} \in \Pi$ fix $\{1\}$.

At last suppose that $\Upsilon$ consists of only one block, i.e. $g=2$, and let $h, k$ respectively be the least and the greatest index of an element in $\Upsilon$. Denote $v_{1}=a_{1} \ldots a_{h-1}$ and $v_{2}=a_{k+1} \ldots a_{t}$, then $a_{h} v_{1} a_{h}, a_{k} v_{2} a_{k}$ are factors of $w$ whence $1 v_{1}, 1 v_{2} \in\{1,2\}$ are equations occurring in $\Gamma_{w}(\Pi, \Upsilon)$.

In this case, suppose first that $1 v_{2}=1$. Since for all $a_{i} \in \Sigma, a_{k} v_{2} a_{i} v_{1} a_{h}$ and $a_{k} v_{2} a_{i}^{2} v_{1} a_{h}$ are factors of $w$, equations in $\Gamma_{w}(\Pi, \Upsilon)$ give $1 a_{i} v_{1} \in\{1,2\}$ and $1 a_{i}^{2} v_{1} \in\{1,2\}$. Moreover since $w$ contains $a_{k} a_{i} a_{k}$ or $a_{k} v_{2} a_{i} a_{k} a_{i}$ we have $1 a_{i} \in\{1,2\}$ for every $a_{i} \in \Sigma$. If all the letters $a_{i}$ fix 1 , then we are done. Otherwise suppose there is a letter $a_{i}$ such that $1 a_{i}=2$. Then we get $2 v_{1} \in\{1,2\}$ and since $1 v_{1} \in\{1,2\}$ we obtain that $v_{1}$ fixes $\{1,2\}$. Thus from $1 a_{i}^{2} v_{1} \in\{1,2\}$ we get $2 a_{i}=1$ and $a_{i}=(12) \ldots$ To prove that in this case all permutations are of the same type, note that whenever a factor $a_{k} v_{2} a_{i} a_{j} a_{i} v_{1} a_{h}$ appears in $w$ we have $1 a_{i} a_{j} a_{i} \in\{1,2\}$ and then apply Lemma 4

Suppose now $1 v_{2}=2$. Then $2 a_{i} v_{1}, 2 a_{i}^{2} v_{1} \in\{1,2\}$ for every $a_{i} \in \Pi$. Let us prove that $v_{1}$ must fix $\{1,2\}$. This is trivial if $h=1$ and $v_{1}$ is empty, else let $\{1,2\}=\left\{1 v_{1}, z v_{1}\right\}$ for some $z$ and prove that $z=2$. For all $i: 1 \leq i<h a_{k} v_{2} a_{i} a_{h} a_{i}$ and $a_{i} a_{h} a_{i} v_{1} a_{h}$ are factors of $w$, hence $1 v_{2} a_{i} \in\{1,2\}$ and $1 a_{i} v_{1} \in\{1,2\}$ are equations in $\Gamma_{w}(\Pi, \Upsilon)$, so that $2 a_{i} \in\{1,2\}$ and $a_{i} v_{1}$ fixes $\{1,2\}$. If for some $i: 1 \leq i<h: 2 a_{i}=2 a_{i}^{2}$ then $a_{i}=(2) \ldots$ and $2 v_{1} \in\{1,2\}$ whence $z=2$; else $\left\{2 a_{i}, 2 a_{i}^{2}\right\}=\{1, z\}$ which immediately yields $a_{i}=(21 z \ldots) \ldots$ since $2 \neq 2 a_{i} \in\{1,2\}$. If there exist two such elements $a_{i}, a_{j}, 1 \leq i<j<h$ then the factor $a_{k} v_{2} a_{i} a_{j} a_{i} v_{1} a_{h}$ occurs in $w$ so that $1 v_{2} a_{i} a_{j} a_{i} v_{1}=\left(2 a_{i} a_{j}\right) a_{i} v_{1} \in$ $\{1,2\}$ is an equation in $\Gamma_{w}(\Pi, \Upsilon)$ whence $2 a_{i} a_{j}=z \in\{1,2\}$ and $z=2$; but if only one element $a_{i}$ of this type exists then $z=1 a_{i}=1 v_{1} \in\{1,2\}$. Thus $v_{1}$ fixes $\{1,2\}$, which immediately yields $2 a_{i}, 1 a_{i} \in\{1,2\}$ for every $a_{i} \in \Pi, i<k$ by the occurrence of the factor $a_{k} v_{2} a_{i} a_{k} a_{i} v_{1} a_{h}$, and for every $a_{i} \in \Pi, i>k$ by the occurrence of the factors $a_{k} a_{i} a_{k}$ and $a_{k} v_{2} a_{i} v_{1} a_{h}$; hence all elements in $\Pi$ fix $\{1,2\}$ so that they are special of the same type.

Thus $w$ is 2-collapsing because the systems associated to $w$ for each role assignment $(\Pi, \Upsilon)$ have only trivial solutions and the length of the word $w$ is:

$$
t+t(t+1)+t(t+2)+\frac{t(t-1)}{2}(t+3)=\frac{t^{3}+6 t^{2}+5 t}{2} .
$$




\section{Short 2-collapsing words.}

The bound found in Theorem 6 is lower than the one given in [9], but still for small values of $n$ it reveals to be not efficient: indeed for $n=3,4,5$ it respectively gives $|w| \leq 48,90,150$. It was proved in [3] that the shortest 2-collapsing words on 3 letters have length 21 and the shortest 2-synchronizing words on 3 letters have length 20 , while a 2-collapsing word on 4 letters is known whose length equals 58 ([4]). But we can use our lemmas to get shorter 2-collapsing and 2-synchronizing words on 4 and 5 letters.

We will make a systematic use of the following

Lemma 7 Let $w$ be a word on an alphabet $\Sigma$ with $|\Sigma| \geq 2$, let $\Sigma^{\prime} \subseteq \Sigma$ and $w^{\prime} \in \Sigma^{\prime+}$ be a factor of $w$, which is a 2-collapsing word on $\Sigma^{\prime}$. For a role assignment $(\Pi, \Upsilon)$ of $\Sigma$ let $\Pi^{\prime}=\Pi \cap \Sigma^{\prime}$ and $\Upsilon^{\prime}=\Upsilon \cap \Sigma^{\prime}$ and for each block $E_{i}\left(D_{i}\right)$ in $\Upsilon$ let $E_{i}^{\prime}=E_{i} \cap \Sigma^{\prime}\left(D_{i}^{\prime}=D_{i} \cap \Sigma^{\prime}\right)$. Then for each role assignment $(\Pi, \Upsilon)$ such that $\emptyset \neq \Pi^{\prime}$ and $\emptyset \neq \Upsilon^{\prime}$ the following properties hold:

- If $(\Pi, \Upsilon)$ is a MONO-role assignment with $\Upsilon=D_{2}$, then in each solution of $\Gamma_{w}(\Pi, \Upsilon)$ the elements in $\Pi^{\prime}$ are special of the same type with respect to $(1,2)$.

- If $(\Pi, \Upsilon)$ is a a MONO-role assignment with $\Upsilon=\left\{D_{2}, D_{3}, \ldots D_{g}\right\}, g>2$, and there are at least two non empty blocks $D_{i_{1}}^{\prime}, D_{i_{2}}^{\prime}$, then in each solution of $\Gamma_{w}(\Pi, \Upsilon)$ all the elements in $\Pi^{\prime}$ fix $\{1\}$.

- If $(\Pi, \Upsilon)$ is a STEREO-role assignment and both $E_{1}^{\prime}, E_{2}^{\prime}$ are nonempty, then in each solution of $\Gamma_{w}^{\prime}(\Pi, \Upsilon)$ all the elements in $\Pi^{\prime}$ fix the set $\{1,2\}$.

In all the above cases, if $\Pi^{\prime}=\Pi$ then the system associated to $w$ for $(\Pi, \Upsilon)$ has only trivial solutions.

Proof: If $(\Pi, \Upsilon)$ is a MONO-role assignment, then $\left(\Pi^{\prime}, \Upsilon^{\prime}\right)$ is a MONO-role assignment of $\Sigma^{\prime}$ and the equations of $\Gamma_{w^{\prime}}\left(\Pi^{\prime}, \Upsilon^{\prime}\right)$ occur in $\Gamma_{w}(\Pi, \Upsilon)$, hence all solutions of the latter system are also solutions of the former. Hence if $\Upsilon$ and consequently $\Upsilon^{\prime}$ has only one block, then all elements in $\Pi^{\prime}$ are special permutations relative to $(1,2)$ which all fix $\{1\}$ or all fix $\{1,2\}$, because $w^{\prime}$ is 2-collapsing. Similarly if $\Upsilon^{\prime}$ is formed by at least two blocks then all elements in $\Pi^{\prime}$ fix $\{1\}$. If $(\Pi, \Upsilon)$ is a STEREO-role assignment of $\Sigma$, and $\Upsilon^{\prime}$ has two non trivial blocks, then $\left(\Pi^{\prime}, \Upsilon^{\prime}\right)$ is a STEREO-role assignment of $\Sigma^{\prime}$ hence the equations of $\Gamma_{w^{\prime}}^{\prime}\left(\Pi^{\prime}, \Upsilon^{\prime}\right)$ occur in $\Gamma_{w}^{\prime}(\Pi, \Upsilon)$ and so all elements in $\Pi^{\prime}$ are special permutations relative to $(1,2)$ which all fix $\{1,2\}$. The last statement is trivial.

It is important to remark, using the same notation and the same arguments of the above Lemma 7, that if $(\Pi, \Upsilon)$ is a MONO-role assignment with more than one block different from $\Pi$, while $\Upsilon^{\prime}$ is formed by a unique block $D_{i}^{\prime}$, then in each solution of $\Gamma_{w}(\Pi, \Upsilon)$ all the elements in $\Pi^{\prime}$ are special of the same type with respect to $\{1, i\}$. Similarly if $(\Pi, \Upsilon)$ is a STEREO-role assignment of $\Sigma$ and $E_{1}^{\prime}=\emptyset$ then in each solution of $\Gamma_{w}^{\prime}(\Pi, \Upsilon)$ all the elements in $\Pi^{\prime}$ are special of the same type with respect to $(2,1)$ while if $E_{2}^{\prime}=\emptyset$ then all the elements in $\Pi^{\prime}$ are special of the same type with respect to $(1,2)$.

\section{Proposition 8}

$$
c(2,4) \leq 56
$$

Proof: Let $\Sigma=\{a, b, c, d\}$ and let

$$
\begin{aligned}
r & =c a b a c^{2} b a c b c a c b^{2} a^{2} c \\
s & =d a d b d a^{2} b^{2} d a b d^{2} a b \\
u & =a d a c d b c a d a c^{2} d^{2} c b d c a .
\end{aligned}
$$


Consider the word $w=r(b a b) s u$. Of course $|w|=56$; we want to prove that $w$ is 2-collapsing. In order to exhibit $w$ we made use of two of the minimal 2-collapsing words on 3 letters. Namely $u_{1}=r(b a b)$ is the 55-th word in the list in [3] on the alphabet $\{c, a, b\}$, while $u_{2}=(b a b) s(a d)$ is the 11-th one in that list on the alphabet $\{b, a, d\}$. Both the words $u_{1}$ and $u_{2}$ are 2-collapsing, as shown in [3]. Put $\Sigma_{1}=\{a, b, c\}$ and $\Sigma_{2}=\{a, b, d\}$.

Let us consider different role assignments $(\Pi, \Upsilon)$ of $\Sigma$ and put $\Pi_{i}=\Pi \cap \Sigma_{i}, \Upsilon_{i}=\Upsilon \cap \Sigma_{i}$, for $i=1,2$.

Let $|\Pi|=1$ and $\Pi \subset\{a, b\}$ then $\Pi_{1}=\Pi_{2}=\Pi$. Moreover if $(\Pi, \Upsilon)$ induces on $\Upsilon$ a partition in more than one block then there is at least an $i=1,2$ such that $\Upsilon_{i}$ contains two non empty blocks, so the system associated to $w$ for $(P, \Upsilon)$ has only trivial solutions by Lemma 7 Then let $\Pi=\{c\}$. If $(\Pi, \Upsilon)$ is a MONO-role assignment and $\Upsilon$ consists of only one block, then all the solutions of $\Gamma_{w}(\Pi, \Upsilon)$ are trivial by Lemma 7. If $\Upsilon$ consists of more than one block then one has to consider the factors $a c b, b c a$ of $w$ if the letters $a$ and $b$ belong to different blocks, else consider the factors $a c d$ and $d c a$. In both cases, if $(\Pi, \Upsilon)$ is a MONO-role assignment then the equations $1 c \in\{1,2\}, 1 c \in\{1,3\}$ occur in $\Gamma_{w}(\Pi, \Upsilon)$ hence $c$ fixes $\{1\}$. If $(\Pi, \Upsilon)$ is a STEREO-role assignment then the equations $1 c \in\{1,2\}, 2 c \in\{1,2\}$ occur in $\Gamma_{w}^{\prime}(\Pi, \Upsilon)$, hence $c$ fixes the set $\{1,2\}$. The same argument applies when $\Pi=\{d\}$, considering the factors $a d b, b d a$ or $b d c, c d b$.

Let $|\Pi|=2$. Assume $\Upsilon=\{a, b\}$. Then for all $i=1,2, \emptyset \neq \Pi_{i} \neq \Pi$ and $\Upsilon=\Upsilon_{i}$. Then if $\Upsilon$ is formed by two blocks then all the solutions of the system associated to $w$ for $(\Pi, \Upsilon)$ are trivial: by Lemma 7 if $(\Pi, \Upsilon)$ is a MONO-role assignment then both $c, d$ fix $\{1\}$, if $(\Pi, \Upsilon)$ is a STEREO-role assignment then both $c, d$ fix $\{1,2\}$. Otherwise if $\Upsilon$ is formed by a unique block we only get that $c$ and $d$ are special permutations relative to $(1,2)$. But since $w$ contains the factors $a c d b, b d c a$, the equations $1 c d \in\{1,2\}, 1 d c \in\{1,2\}$ occur in $\Gamma_{w}(\Pi,\{a, b\})$ hence $c, d$ are special permutations of the same type with respect to $(1,2)$, i.e. the system $\Gamma_{w}(\Pi,\{a, b\})$ has only trivial solutions. Then let $\Upsilon \neq\{a, b\}$, whence $\Pi=\Pi_{i}$ for some $i=1,2$. Hence if $\Upsilon$ is formed by a unique block then $\Gamma_{w}(\Pi, \Upsilon)$ has only trivial solutions by Lemma7 7 Otherwise we only get that the solutions of the systems associated to $w$ for $(\Pi, \Upsilon)$ are either special permutations relative to $(1,2)$ or fix $\{2\}$. Denote by $\beta_{1}, \beta_{2}$ the elements in $\Pi$ and by $\alpha_{1}, \alpha_{2}$ the elements in $\Upsilon$; for every choice of $(\Pi, \Upsilon)$ where $\Upsilon \neq\{a, b\}$ is formed by two blocks, there is an $i=1,2$ such that $\alpha_{h} \beta_{i} \alpha_{h}$ and either $\alpha_{h} \beta_{i}^{\epsilon} \beta_{3-i} \alpha_{h}$ or $\alpha_{h} \beta_{i}^{\epsilon} \beta_{3-i} \alpha_{3-h}$ (where $\epsilon \in\{0,1\}$ and $\beta_{i}^{0}$ is the empty word) are factors of $w$ for all $h=1,2$. Then if $(\Pi, \Upsilon)$ is a STEREO-role assignment then the equations $1 \beta_{i} \in\{1,2\}, 2 \beta_{i} \in\{1,2\}, 1 \beta_{i}^{\epsilon} \beta_{3-i} \in\{1,2\}, 2 \beta_{i}^{\epsilon} \beta_{3-i} \in\{1,2\}$ occur in $\Gamma_{w}^{\prime}(\Pi, \Upsilon)$, and this yields that the solutions of the systems are pairs of special permutations which both fix the set $\{1,2\}$, i.e. the system has only trivial solutions. Otherwise if $(\Pi, \Upsilon)$ is a MONO-role assignment with three blocks the equations $1 \beta_{i} \in\{1,2\}, 1 \beta_{i} \in\{1,3\}, 1 \beta_{i}^{\epsilon} \beta_{3-i} \in\{1,2\}, 1 \beta_{i}^{\epsilon} \beta_{3-i} \in\{1,3\}$ occur in $\Gamma_{w}(\Pi, \Upsilon)$, and this yields that the solutions of the systems are pair of trivial permutations which both fix $\{1\}$, hence again the system has only trivial solutions.

Let $|\Pi|=3$. If $\Upsilon=\{\alpha\} \subset\{a, b\}$ then for all $i=1,2 \Upsilon_{i}=\Upsilon$, hence the solutions of $\Gamma_{w}(\Pi, \Upsilon)$ are triples of special permutations of the same type of $\gamma \in\{a, b\}-\Upsilon$, since they are also solutions of $\Gamma_{u_{i}}\left(\Pi_{i}, \Upsilon_{i}\right)$. If these permutations are not all of the same type then it must be $\gamma=(1)(2) \ldots$. Then if $\gamma=b$ consider the factor $a c d b c a$ of $w$, otherwise consider the factor badacdb of $w$. In the first case the equation $1 c d b c \in\{1,2\}$ occurs in $\Gamma_{w}(\Pi, \Upsilon)$, in the second case 1adacd $\in\{1,2\}$ occurs in $\Gamma_{w}(\Pi, \Upsilon)$, and both the equations together with $\gamma=(1)(2) \ldots$ yield that $c, d$ are of the same type. Hence the system $\Gamma_{w}(\Pi, \Upsilon)$ has only trivial solutions.

If $\Upsilon=\{c\}$ then $a, b$ are special permutations of the same type with respect to $(1,2)$ since they are solutions of $\Gamma_{u_{1}}(\{a, b\},\{c\})$, whence $1 a, 1 a^{2}, 1 b, 1 b^{2} \in\{1,2\}$ and $b, d$ are special solutions of the same 
type with respect to $(1,2)$ by applying Lemma 5 to $\Gamma_{u}(\{a, b\},\{c\}) \cup\left\{1 b, 1 b^{2} \in\{1,2\}\right\}$. Whence $1 d, 1 d^{2} \in\{1,2\}$ and again $a, d$ are special solutions of the same type with respect to $(1,2)$ by applying Lemma 4 to

$$
\Gamma_{u}(\{a, b\},\{c\}) \cup\left\{1 a, 1 a^{2}, 1 d, 1 d^{2} \in\{1,2\}\right\} .
$$

If $\Upsilon=\{d\}$, then $a, b$ are special solutions of the same type with respect to $(1,2)$ since they are solutions of $\Gamma_{u_{2}}(\{a, b\},\{d\})$, whence $1 a, 1 a^{2}, 1 b, 1 b^{2} \in\{1,2\}$. More $1 a c, 1 b c a, 1 a c^{2}, 1 c b \in\{1,2\}$ by $\Gamma_{u}(\{a, b\},\{d\})$. If $a$ fixes $\{1\}$ but does not fix $\{2\}$, then $b$ too fixes $\{1\}$ and we immediately get $1 a c, 1 c a, 1 c^{2}, 1 c \in\{1,2\}$ whence $a$ and $c$ are special solutions of the same type with respect to $(1,2)$ by applying Lemma 5 so that also $c$ fixes $\{1\}$. If $a=(1)(2) \ldots$ then $1 c, 1 b c, 1 c^{2}, 1 c b \in\{1,2\}$ whence again $b$ and $c$ are trivial solutions of the same type by Lemma 5 Finally, let $a=(12) \ldots$; then $b$ fixes $\{1,2\}$ whence $2 c, 1 c \in\{1,2\}$.

\section{Proposition 9}

$$
c(2,5) \leq 119
$$

Proof: Let $\Sigma=\{a, b, c, d, e\}$ and let

$$
\begin{aligned}
& r=c a b a c^{2} b a c b c a c b^{2} a^{2} c, \\
& s=d a d b d a^{2} b^{2} d a b d^{2} a b, \\
& u=a d a c d b c a d a c^{2} d^{2} c b d c a \text {, } \\
& s^{\prime}=\text { abeaebea } a^{2} b^{2} e a b e^{2} a b, \\
& u^{\prime}=\text { aeacebcaeac }{ }^{2} e^{2} \text { cbe. }
\end{aligned}
$$

Consider the words $w_{1}=r(b a b) s u \in\{a, b, c, d\}^{+}$and $w_{2}=b s^{\prime} u^{\prime} r(b a b) \in\{a, b, c, e\}^{+}$.

Like in the proof of Proposition 8 the word $w_{1}$ is 2-collapsing on the letters $\{a, b, c, d\}$ and symmetrically the word $w_{2}$ is 2-collapsing on the letters $\{a, b, c, e\}$ : we only changed the order of some parts, but still we involved as factors some of the minimal 2-collapsing words on three letters. The factors in $u$ and $u^{\prime}(\mathrm{ca})$ strictly correspond to the one we considered in the proof of Proposition 8 .

More, let $v=$ cedbeacdebdedbedade $d^{2}$ ecedce and finally $w=s^{\prime} u^{\prime} r(b a b) s u v$.

(Note that $w$ contains all the factors of $w_{2}$ ). Of course $|w|=119$; we want to prove that $w$ is 2-collapsing. Let us consider different role assignments $(\Pi, \Upsilon)$ of $\Sigma$ and put $\Pi_{i}=\Pi \cap \Sigma_{i}, \Upsilon_{i}=\Upsilon \cap \Sigma_{i}$, for $i=1,2$.

If $|\Pi|=1$ arguments like the ones applied in the proof of Proposition 8 can be used to prove that the system associated to $w$ for $(\Pi, \Upsilon)$ has only trivial solutions.

Let $|\Pi|=2,3$. Assume that, for some $i=1,2, \Pi \subseteq \Sigma_{i}$. If $\Upsilon$ is formed by a unique block, then the system associated to $w$ for $(\Pi, \Upsilon)$ has only trivial solutions by Lemma 7 , the same happens when both $\Upsilon_{i}$ and $\Upsilon$ are decomposed in more than one block. So, for all $i$ such that $\Pi \subseteq \Sigma_{i}, \Upsilon_{i}$ is formed by a unique block while $\Upsilon$ is formed by more than one block. It is easy to check that if $\Pi \subset\{a, b, c\}$ then either $\Upsilon_{1}$ or $\Upsilon_{2}$ are formed by two blocks. Then assume $\Pi=\{a, b, c\}$, whence $\Upsilon=(\{d\},\{e\})$. Hence all the solutions of the system associated to $w$ for $(\Pi, \Upsilon)$ fix $\{1,2\}$ by the remark after Lemma 7 Assume that $\Pi$ is not a subset of $\{a, b, c\}$ and $\Pi \subseteq \Sigma_{1}$, then $\Pi_{1}=\Pi$ and $\Upsilon_{2}=\Upsilon=\left(\Upsilon_{1},\{e\}\right)$. In all the solutions of the system associated to $w$ for $(\Pi, \Upsilon)$ by Lemma 7 the elements in $\Pi_{2}$ fix $\{1,2\}$ if $(\Pi, \Upsilon)$ is a STEREO-role assignment and fix $\{1\}$ if it is a MONO-role assignment. Moreover each solution of the system associated with $w$ for $(\Pi, \Upsilon)$ is formed by permutations which either fix $\{1,2\}$ or fix $\{1\}$ or fix $\{2\}$ (by remark after 
Lemma 7). So let $(\Pi, \Upsilon)$ be a STEREO-role assignment. Then the solutions of the system $\Gamma_{w}^{\prime}(\Pi, \Upsilon)$ either fix all $\{1,2\}$, or all fix $\{1\}(\{2\})$ and the elements in $\Pi_{2}$ are of the form $(1)(2) \ldots$ In the latter case, since edbe, edce are factors of $w$, when either $b$ or $c$ are in $\Pi_{2}$ we get that $d$ too has the form $(1)(2) \ldots$, otherwise we get again that $d$ has the form (1)(2)... because $w$ has the factors $e d b, e d c$. Hence $\Gamma_{w}^{\prime}(\Pi, \Upsilon)$ has only trivial solutions. Then let $(\Pi, \Upsilon$ ) be a MONO-role assignment (with more than one block in $\Upsilon$ ). Since $w$ contains all the factors ade, bde, cde we get that $d$ fixes $\{1\}$, whence $\Gamma_{w}(\Pi, \Upsilon)$ has only trivial solutions. Similar arguments apply when $\Pi$ is not a subset of $\{a, b, c\}$ and $\Pi \subseteq \Sigma_{2}$. So let $\{d, e\} \subseteq \Pi$. Obviously $\Upsilon_{i}=\Upsilon$ for all $i=1,2$. Then if $\Upsilon$ contains at least two blocks then the solutions of the system associated to $w$ for $(\Pi, \Upsilon)$ are trivial by Lemma 7. So let $(\Pi, \Upsilon)$ be a MONO-role assignment with a unique block in $\Upsilon$. Then all the solutions of $\Gamma_{w}(\Pi, \Upsilon)$ are formed by special permutations with respect to $(1,2)$ and if $\left|\Pi_{i}\right|>1$ then the permutations in $\Pi_{i}$ are of the same type. Assume that the permutations in $\Pi_{1}$ and in $\Pi_{2}$ are not of the same type then if $\Pi \subseteq\{a, d, e\}$, the factors $c d e b, b d e c, c e d b$, bedc give that both $d, e$ fix $\{1,2\}$. Moreover beaeb is a factor of $w$ so again both $a, e$ fix $\{1,2\}$ provided that $a \in \Pi$. Now let $\Pi=\{b, d, e\}$. If there is a solution of $\Gamma_{w}(\Pi, \Upsilon)$ which is not trivial then $b$ has the form $(1)(2) \ldots$, and the factor cedbea gives that both $d, e$ fix $\{1,2\}$. If $\Pi=\{c, d, e\}$ then if there is a solution of $\Gamma_{w}(\Pi, \Upsilon)$ which is not trivial then $c$ has the form (1)(2)..., and again the factor bdedb gives that both $d$ and $e$ fix $\{1,2\}$. Hence the systems associated to $w$ for each partition $(\Pi, \Upsilon)$ with $|\Pi|=2,3$ have only trivial solutions.

So, let $|\Pi|=4$ and let $\Upsilon=\{\alpha\}$. If $\alpha \in\{a, b, c\}$ then in each solution of $\Gamma_{w}(\Pi, \Upsilon)$ all the elements in $\Pi_{1}$ are special permutations of the same type with respect to $(1,2)$ and the same holds for all the elements in $\Pi_{2}$. So if in $\Pi$ there exist elements of different form then the elements in $\{a, b, c\}-\{\alpha\}$ must have the form $(1)(2) \ldots$ But, in such case, considering the equations generated by some of the factors acedbea, bdcacedb, cedbeac of $w$ it is easy to verify that also $d$ and $e$ fix $\{1,2\}$. If $\alpha=d$ then in each solution of $\Gamma_{w}(\Pi, \Upsilon) a, b, c$ are special of the same type with respect to $(1,2)$ by Lemma 7 and 1 cace, 1 beac, 1 eb, $1 e, 1 b e, 1 e^{2}$, 1ece $\in\{1,2\}$ occur in $\Gamma_{(b d c a) v}(\Pi, \Upsilon)$ whence they occur also in $\Gamma_{w}(\Pi, \Upsilon)$; hence $b, e$ and $c, e$ are special of the same type with respect to $(1,2)$. If one among $b, c$ is different from (1)(2)... then all of them are of the same type; but if both of them fix both 1 and 2 then one gets $1 a e, 1 e a \in\{1,2\}$ and so all the solutions of $\Gamma_{w}(\Pi, \Upsilon)$ are trivial. If $\alpha=e$ then again in each solution of $\Gamma_{w}(\Pi, \Upsilon) a, b, c$ are special of the same type with respect $(1,2)$. Moreover $1 d b, 1 a c d, 1 b d, 1 d a d, 1 d^{2}, 1 d c \in\{1,2\}$ occur in $\Gamma_{(b d c a) v}(\Pi, \Upsilon)$, whence $d, b$ and $d, a$ are special of the same type by Lemmas 5 and 4 Again, assume $a=b=(1)(2) \ldots:$ this yields at last $1 c d, 1 d c \in\{1,2\}$ and again all the solutions of $\Gamma_{w}(\Pi, \Upsilon)$ are trivial.

In Proposition 8 of [7] we considered a slightly longer word, so proving that $c(2,5) \leq 120$. But in [11] an algorithm is presented to find shorter 2-collapsing words from a given 2-collapsing word. The author run her algorithm on our word and got four 2-collapsing words of length 119, one of which is the word considered in the above Proposition 9. She also did the same on the word in our Proposition 8, she could not find shorter 2-collapsing words, but found three more 2-collapsing words of the same length.

Of course Propositions 8 and 9 give upper bounds to the length of the minimal 2-synchronizing words respectively on 4 and 5 letters. But this bounds can be further improved: denote by $s(2, t)$ the length of the least 2 -synchronizing word on $t$ letters.

\section{Proposition 10}

$$
s(2,4) \leq 52
$$


Proof: Let $\Sigma=\{a, b, c, d\}$ and let

$$
\begin{aligned}
& r=c a b a b^{2} c b c a c a^{2} b^{2} c^{2} a \in\{a, b, c\}^{+} \\
& s=b d^{2} a^{2} b^{2} d b d a d a^{2} b a b d \in\{a, b, d\}^{+}
\end{aligned}
$$

The word $w=\left(c d^{2} c b c d\right) r(a b) s\left(c d a d c^{2} d\right)$ is 2-synchronizing and $|w|=52$. In order to exhibit $w$ we used two of the minimal 2-synchronizing words on 3 letters listed in [3]. Namely $u_{1}=r(a b)$ is the 11-th word in the list in [3], on the alphabet $\Sigma_{1}=\{c, a, b\}$, while $u_{2}=(a b) s$ is the 15-st one in that list, on the alphabet $\Sigma_{2}=\{a, b, d\}$. Both the words $u_{1}$ and $u_{2}$ are 2-collapsing ([3]).

Let us consider different role assignments $(\Pi, \Upsilon)$ of $\Sigma$ and put $\Pi_{i}=\Pi \cap \Sigma_{i}, \Upsilon_{i}=\Upsilon \cap \Sigma_{i}$, for $i=1,2$. The cases when $|\Pi|=1,2$ can be proved with similar arguments as in the proof of Proposition 8 .

Let $|\Pi|=3$. We are looking for solutions of our systems of equations on 3 permutations $t, y, z$ in the symmetric group $\mathrm{Sym}_{3}$. If a system has only special solutions with respect to $\{1,2\}$ and $t, y$ are of different types while both of them are of the same of $z$, then without loss of generality $t=(12)(3)$ and $y=(1)(23)$; in such a case we immediately get that $z=(1)(2)(3)=t^{2}=y^{2}$.

Now let $\alpha \in \Upsilon$. If $\alpha=a$ (resp. $\alpha=b$ ) the permutations $b, c$ (resp. $a, c$ ) are trivial solutions of $\Gamma_{u_{1}}\left(\Pi_{1},\{\alpha\}\right)$ and $b, d$ (resp. $\left.a, d\right)$ are trivial solutions of $\Gamma_{u_{2}}\left(\Pi_{2},\{\alpha\}\right)$, whence both $c$ and $d$ are of the same type as $b$ (resp. of $a$ ). If $b$ (resp. $a$ ) is different from the identity, then both $c$ and $d$ must be of the same type by the previous remark, but this is true also on the other case as the equation $1 b d c d \in\{1,2\}$ belongs to $\Gamma_{w}(\Pi,\{a\})$ (resp. $1 c d c a \in\{1,2\}$ belongs to $\Gamma_{w}(\Pi,\{b\})$ ). If $\alpha=c$ then $a, b$ are trivial solutions of $\Sigma_{u_{1}}\left(\Pi_{1},\{\alpha\}\right)$. More $c d c, c d^{2} c, c d a d c$ are factors of $w$. Since $1 a, 1 a^{2} \in\{1,2\}$ then only special permutations of the same type are solutions of $\Gamma_{w}(\Pi,\{c\})$ by Lemma 4 Thus $b$ and $d$ are special permutations of the same type as $a$. But the equation $1 a u_{2} \in\{1,2\}$ appears in $\Gamma_{w}(\Pi,\{c\})$ : if $a$ is the identity it immediately yields that $1 d b d \in\{1,2\}$ by previous remark. By Lemma $4 b$ and $d$ are of the same type. A similar argument runs when $\alpha=d$ since $d c d, d c^{2} d, d c b c d, d u_{1} b d$ are factors of $w$.

\section{Proposition 11}

$$
s(2,5) \leq 111
$$

Proof: Let $\Sigma=\{a, b, c, d, e\}$. We want to exhibit a 2-synchronizing word of length 111 on $\Sigma$, like in the proof of Proposition 10 We shall consider two slightly longer 2-synchronizing word on 4 letters with a better overlapping:

$$
\begin{gathered}
r=a b^{2} c^{2} a b a c a b^{2} c b c a^{2} c ; s=b d^{2} a b a d a b a^{2} b d b d^{2} a d ; u=a_{d c d^{2} c^{2} d a b c a d c b d} \\
w_{1}=u r(a b) s ; s^{\prime}=s ; r^{\prime}=\text { dedea eadaead }{ }^{2} e^{2} a ; u^{\prime}=\text { dbaebdeabe }^{2} b^{2} e d b \\
w_{2}=(a b) s r^{\prime} u^{\prime} ; v=\text { cebeacdaece } ; v^{\prime}=c e c^{2} e^{2} \text { cbcedec. }
\end{gathered}
$$

The word $w_{1}$ (resp. $w_{2}$ ) is 2-synchronizing on the alphabet $\{a, b, c, d\}$ (resp. $\{a, b, d, e\}$ ), by similar arguments as in the proof of Proposition 10. In fact it is built from two words in the list in [3] for suitable alphabets: $r(a b)$ is the 6-th and $(a b) s$ is the 16-th (resp. $(a b) s^{\prime}$ is the 16-th while $(d a d) r^{\prime}$ is the 1-st) and $u$ (resp. $u^{\prime}$ ) gives us the other necessary factors.

The word $w=\operatorname{vur}(a b) s r^{\prime} u^{\prime} v^{\prime}$ is 2-synchronizing and its length equals 111 . 


\section{Acknowledgements}

The authors thank Elena Pribavkina for informing them about the results of applying her algorithm on the short 2-collapsing words presented in [7] and for her careful reading of the present paper.

\section{References}

[1] D. S. Ananichev, A. Cherubini, M. V. Volkov, Image reducing words and subgroups of free groups, Theor. Comput. Sci. 307, no.1, (2003), 77-92.

[2] D. S. Ananichev, A. Cherubini, M. V. Volkov, An inverse automata algorithm for recognizing 2collapsing words, in: M. Ito, M. Toyama (eds.), Developments in Language Theory, Lect. Notes Comp. Sci. 2450, Springer, Berlin 2003, 270-282.

[3] D. S. Ananichev, I. V. Petrov, Quest for short synchronizing words and short collapsing words, WORDS 2003. Proc. 4th Int. Conf., Univ. of Turku, Finland, 411-418.

[4] D. S. Ananichev, I. V. Petrov, M. V. Volkov, Collapsing words: a progress report, Internat. J. Foundations Comp. Sci. 17, no.3, (2006), 507-518.

[5] J. Černý, Poznámka k homogénnym eksperimentom s konečnými automatami, Mat.-Fyz. Cas. Slovensk. Akad. Vied. 14, (1964), 208-216 [in Slovak].

[6] A. Cherubini, Synchronizing and collapsing words, Milan J. Math. 75, (2007), 305-321.

[7] A. Cherubini, A. Kisielewicz, B. Piochi, A bound for the length of shortest 2-collapsing words, in: P. Arnoux, N. Bedaride, J. Cassaigne (eds.), WORDS 2007. Proc. 6th Int. Conf. on Words, 90-99.

[8] A. Cherubini, P. Gawrychowski, A. Kisielewicz, B. Piochi, A combinatorial approach to collapsing words, in: R. Krýlovič, P. Urzyczyn (eds.), Mathematical Foundations of Computer Science 2006, Lect. Notes Comp. Sci. 4162, Springer, Berlin 2006, 256-266.

[9] S. W. Margolis, J.-E. Pin, M. V. Volkov, Words guaranteeing minimum image, Internat. J. Foundations Comp. Sci. 15, no.2, (2004), 259-276.

[10] E. V. Pribavkina, On some properties of the language of 2-collapsing words, Internat. J. Foundations Comp. Sci. 17, no.3, (2006), 665-676.

[11] E. V. Pribavkina, 2-collapsing words and a sequence reconstruction problem, CD Proceedings of AutoMathA Conference 2007, Mondello, Italy.

[12] N. Sauer, M. G. Stone, Composing functions to reduce image size, Ars Combinatoria 31, (1991), $171-176$. 